\title{
An argument for a rational and balanced risk approach to transplantation during the COVID-19 pandemic
}

\author{
L Brannigan, J Botha \\ Wits Transplant, Wits Donald Gordon Medical Centre, South Africa \\ Corresponding author, email:jfbwdgmc@gmail.com
}

Since the December 2019 discovery of the SARS-CoV-2, the COVID-19 pandemic has impacted healthcare systems globally. Worldwide, even the most advanced and wellfunded healthcare systems have been found wanting with respect to their readiness to manage the pandemic and also to ensure the ongoing viability of other important health related services. While much of the media and medical community remains focussed on the direct impacts associated with the pandemic and on servicing the millions of patients afflicted with this disease, there is mounting evidence for the need of advocacy for the maintenance of services unrelated to COVID-19 care but which remain critical for millions of patients worldwide. ${ }^{1}$

The death toll from COVID-19 is currently just over a million. While this figure, calculated between 25 Jan 2020 and 29 September 2020, is significant, it remains important to compare it with other causes of death. The total mortality rate from COVID-19 as well as the global mortality statistics for 2017 show that should global COVID-19 infection trajectory continue in the coming months of 2020, and the mortality rate remains similar, the pandemic deaths would be less than any of the 2017 top 10 causes of death (all over 1.2 million) none of which have disappeared during the pandemic. There is evidence that services for patients afflicted by these conditions, and with particular relevance to this article, transplant services, have been severely curtailed. ${ }^{2}$

This article, utilising transplantation as an example, hopes to argue for a more balanced approach to the maintenance of healthcare services during the COVID-19 pandemic. The excess mortality caused through the curtailment of services cannot be ignored and those responsible for the provision of healthcare should be reminded that they have a responsibility to ensure ALL patients receive access to care.

The two fundamental premises on which the argument for the curtailment of non-COVID-19 related healthcare services, such as transplantation, are based on the risk of continuing such services to patients, such as those requiring surgery, and the unavailability of resources such as healthcare workers and beds being utilised for COVID-19 patients.

\section{On the risk}

There has been much discussion regarding the risk of, for example, surgery during the COVID-19 pandemic with numerous publications citing increased mortality rates in patients during the perioperative period. ${ }^{3}$ This, along with the perceived risk of providing ongoing services such as transplantation, is cited as the reason for the discontinuation of these services.

There are several problems with this argument which should be answered.

Much of the data on perioperative mortality in COVID-19 patients are skewed by the fact that the same risk factors for mortality from the virus, not unsurprisingly, are the risk factors that predict mortality from major surgery. Factors such as age over 70, number of comorbidities and emergency surgery are independently strong predictors of perioperative mortality. The vast majority of deaths in the COVID-19 group in numerous studies have some or all of the above risk factors present. ${ }^{4}$ One should be cautious when attributing a causal relationship between the virus and the surgical outcome when these confounders are present. While the perioperative presence of an active viral infection no doubt increases perioperative mortality, one must place this in the context of the need for the intervention.

Mortality from other causes may remain in excess of that of the intervention associated mortality caused by COVID-19 infection. In our local liver transplant programme, the largest of its kind in sub-Saharan Africa, the wait-list mortality for adults was $13 \%$ for 2019 . This has no doubt been rising during the pandemic (our evidence suggests this may approach $20 \%$ !). The lack of available cadaveric donors and the curtailment of services to the pre-transplant patients along with the late referral of cases, all as a result of the COVID-19 pandemic, have resulted in a noticeable increase in mortality. One cannot ignore the mortality associated with untreated cardiovascular diseases, malignancies, transplant treatable conditions and others. This "excess mortality" caused indirectly either by the collateral damage of COVID-19 overwhelming healthcare systems or by the deliberate actions of healthcare administrators prioritising the care of COVID-19 patients cannot and must not be ignored. ${ }^{5}$

Specifically, with respect to transplant patients, there has been much debate regarding the ethics of providing transplant services to patients who may be at risk for 
perioperative mortality. This safety concern is based on the premise that immunocompromised patients may be at greatest risk from demise if they acquire COVID-19. While this is a genuine concern, the argument regarding the fatality rate of their underlying conditions which necessitates transplantation aside, there is very little empiric data to support this notion. Much of the patient data is in patients who had undergone transplantation prior to the pandemic who subsequently contracted the virus which makes the argument for cessation of transplantation services non-sensical. Also, the association between raised IL-6 levels and severity of the pulmonary complications (the commonest cause of death related to COVID-19 infection) point to the fact that immunosuppressive therapies such as corticosteroids have unsurprisingly been shown to be of benefit. There is now emerging data that immunosuppressive therapy may be effective in preventing severe COVID-19 disease and certainly data from the large cancer cohorts are encouraging. 6,7

Lastly, regarding risk, there have been concerns raised that inadvertently transplanting organs from donors with COVID-19 may place recipients at greater risk for mortality, or that transplanting patients who have an unknown COVID-19 infection would equally increase the risk. Numerous transplant programmes have demonstrated that by increased risk assessment, testing donors and recipients preoperatively and adequate perioperative isolation this risk is minimised. There is also literature to support that the inadvertent transplantation of organs from recipients with other viral infections such as influenza has resulted in minimal harm to recipients and there is no good reason to assume this would be different with the COVID-19 outbreak. In fact, the Vanderbilt University experience, with record numbers of transplants performed during the pandemic, is an example of how transplantation can be safely continued. Assuming, based on minimal or no evidence, that curtailment of services is appropriate is dangerous and does not follow the scientific method held up as the gold standard by the healthcare community. ${ }^{8}$

\section{Availability of resources}

It is clear that numerous healthcare systems, especially those in the hot zones for the infection, have struggled to cope with the increasing numbers of patients created by the COVID-19 infection. This has led to the rationing of healthcare services. While it is true that healthcare ethics during a pandemic differ from those which are used under normal circumstances in that the greater good principal trumps individual rights, it is important that principals such as utility, equity, efficiency, the rule of rescue and fairness are applied when allocating resources.

It is concerning that in many countries and regions, there appears to have been a rationing process applied which does not take into account the complexity of the issue, and decisions in this regard seem to be politically influenced and made without the proper consideration. In fact, the Ministerial Advisory Committee (MAC) for transplantation has unilaterally suspended the evaluation of non-related living donor kidney transplant pairs as the committee felt that these transplants should not be taking place (personal communication Professor MR Moosa, chairperson of the MAC). The macro-allocation of resources towards COVID-19 patients is clearly necessary but at the micro- allocation level this may have caused undue denial of access to many non-COVID-19 patients. ${ }^{1-7}$

At a time when hospitals, while seeing an increase in COVID-19 numbers, were not overrun by the pandemic, we have seen numerous transplant and other essential services curtailed. We have witnessed increased mortality, decreasing donor numbers and a decrease in our capacity to manage our patient burden.

Entrenched in the depths of medical ethics are the concepts of the commission of an act and omission. The tendency toward inaction related to the ethical standpoint of non-maleficence is the basis for the common omission bias which occurs in healthcare. This bias, which interestingly occurs more commonly than commission bias, allows for events that are perceived as the "natural course of disease" to be more acceptable than acts attributable directly to the healthcare practitioner. We cannot ignore the inadvertent harm this has caused during the pandemic through the omission to treat non-COVID-19 patients and utilising the need - perceived and demonstrable - for allocation of resources toward COVID-19 positive patients as the rationale for this decision. ${ }^{9}$

One can also no longer ignore the role the media has played in instilling fear into the community and the role that disinformation has played in keeping patients needing care, away from healthcare institutions. The infodemic is a real entity affecting us all and a big driver of public opinion on the pandemic.

What is needed is the rational allocation of resources based on the available scientific knowledge regarding the pandemic, the availability of resources in any given environment and the guidance of the local ethics committee which should have ethicists, clinicians and administrators making decisions. Ensuring care is given to all types of patients to the maximum capacity of the resource is an absolute responsibility of the system.

When confronted with a liver transplant waiting list mortality in our programme approaching $20 \%$ and survival rate after liver transplantation of greater than $85 \%$, can the healthcare system really justify diverting resources away from these patients, particularly when the vast majority of COVID-19 deaths occur in older persons with comorbidities who will, in most cases, have lower short and long term life expectancies? ${ }^{10}$

\section{Summary}

The COVID-19 pandemic has seen an unprecedented strain placed on healthcare systems all over the world. This tragic event has affected all of us and changed the way we deliver and receive healthcare resources. It is important during times like these that we rely on balanced thinking and decision making based on the vast knowledge accumulated in the healthcare community in the modern setting, that media and political influence are kept to a minimum and that we remember the frontline exists for all patients and practitioners, not just those facing the COVID-19 illness directly.

There is a need for a more balanced approach to the delivery of healthcare services to the patient population. We are calling for the recognition that the pandemic is made worse by the excess mortality in non-COVID-19 patients caused by removal of resources for their care and for the need to ensure that essential services are provided to nonCOVID-19 patients. 


\section{References}

1. Angelico R, Trapani S, Manzia TM, et al. The COVID-19 outbreak in Italy: Initial implications for organ transplantation programs. Am J Transplant. 2020;20(7):1780-4.

2. Aron J, Muellbauer J, Giattino C, Ritchie H. A pandemic primer on excess mortality statistics and their comparability across countries [Internet]. 2020. Available from: https:// ourworldindata.org/covid-excess-mortality. Accessed 2020 Jul 18.

3. COVIDSurg Collaborative. Global guidance for surgical care during the COVID-19 pandemic. BJS. 2020;107(9):1097103. Apr 15. https://doi.org/10.1002/bjs.11646.

4. Richards T. An age old problem. Bmj [Internet]. 2007;335(7622):698. Available from: http://www.bmj.com/ cgi/doi/10.1136/bmj.39356.408102.59.

5. Kumar D, Manuel O, Natori Y, et al. COVID-19 : A global transplant perspective on successfully navigating a pandemic.
Am J Transplant. 2020 Jul;20(7):1773-1779. http://doi.org 10.1111/ajt.15876. Epub 2020 Apr 12.

6. Iserson KV. Healthcare ethics during a pandemic. West $\mathrm{J}$ Emerg Med. 2020 Apr 13;21(3):477-483. https://doi.org/10.5 811/westjem.2020.4.47549.

7. Scheunemann LP, White DB. The ethics and reality of rationing in medicine. Chest [Internet]. 2011;140(6):1625-32. https://doi.org/10.1378/chest.11-0622.

8. Cockbain AJ, Jacob M, Ecuyer C, Hostert L, Ahmad N. Transplantation of solid organs procured from influenza A H1N1 infected donors. Transpl Int. 2011 Dec;24(12):e107-10. https://doi.org/10.1111/j.1432-2277.20 11.01342.x. Epub 2011 Sep 29.

9. Hope T. Acts and omissions revisited. J Med Ethics. 2000 Sept;26(4):227-8. https://doi.org/10.1136/jme.26.4.227.

10. Kates OS, Fisher CE, Stankiewicz-Karita HC, et al. Earliest cases of coronavirus disease 2019 (COVID-19) identified in solid organ transplant recipients in the United States. Am J Transplant. 2020;20(7):1885-90. 